\title{
Very early onset and greater vulnerability in schizophrenia: A clinical and neuroimaging study
}

\author{
Francesco Margari' \\ Anna Presicci ${ }^{2}$ \\ Maria Giuseppina \\ Petruzzelli' \\ Patrizia Ventura ${ }^{2}$ \\ Franca Di Cuonzo 3 \\ Michele Palma ${ }^{3}$ \\ Lucia Margari² \\ 'Department of Neurologic \\ and Psychiatry Sciences, Psychiatry \\ Unit; ${ }^{2}$ Department of Neurologic \\ and Psychiatry Sciences, Child \\ Neuropsychiatry Unit; ${ }^{3}$ Department \\ of Neurologic and Psychiatry \\ Sciences, Neuroradiologic Unit, \\ University of Bari, Italy
}

\begin{abstract}
Although schizophrenia has been diagnosed in children, this group of disorders has received too little attention in the clinical and research literature. Preliminary data suggest that early onset schizophrenia (EOS) and very early onset schizophrenia (VEOS) tend to have a worse outcome than adult onset schizophrenia, and seem to be related to a greater familial vulnerability, due to genetic, psychosocial, and environmental factors. Recently, advanced neuroimaging techniques have revealed structural and functional brain abnormalities in some cerebral areas. This paper reports on a case diagnosed as VEOS, with premorbid year-long psychopathological history. The patient showed atypical proton magnetic resonance spectroscopy findings, and normal brain and spine computer tomography and brain magnetic resonance images.

Keywords: very early onset schizophrenia, early onset schizophrenia, premorbid neurodevelopmental abnormalities, magnetic resonance spectroscopy
\end{abstract}

\section{Introduction}

Schizophrenia comprises a group of chronic and debilitating psychiatric disorders, characterized by abnormalities in thinking, perception, and emotion. Schizophrenia is rare in children, affecting about 1 in 1,000, compared to 1 in 100 in adults, with a male/female rate of 1.67 (Campbell et al 1997; Nicolson and Rapoport 1999).

Several studies suggest a clinical continuity between early and later onset schizophrenia (Jacobsen and Rapoport 1998; Nicolson and Rapoport 1999; Asarnow et al 2004). The Diagnostic and Statistical Manual for Mental Disorders, IV Edition (APA 2000) and the International Classification of Disease, X Edition (WHO 1992) propose the same general diagnostic criteria for both forms of schizophrenia. Recently, two classifications have been identified based on the age of onset: early onset schizophrenia (EOS), when the psychotic symptoms are present after the age of 13 years, and very early onset schizophrenia (VEOS), when the onset is at or before the age of 13 years (Werry 1992; Russel 1994). EOS and VEOS show atypical features when compared with adult-onset schizophrenia (AOS): insidious onset; more severe premorbid neurodevelopmental abnormalities; more frequent terrifying visual hallucinations; constant inappropriate or blunted affects; higher rate of familial psychopathology; minor response to treatment; and poorer outcome. Preliminary data suggest that VEOS and EOS may be secondary to greater familial vulnerability, due to genetic, psychosocial, and environmental factors (Werry 1992; Russel 1994; Bailly and de Choudy de Lenclave 2004).

The clinical heterogeneity of VEOS-EOS could be due to the peculiarity of developmental psychopathology, rather than to physiopathological differences between children and adults.

Recent research in neuroscience has improved our understanding of the biological basis of schizophrenia. Advanced neuroimaging techniques applied to psychiatric 
disorders, such as noninvasive proton and phosphorus spectroscopy nuclear magnetic resonance imaging, have defined significant abnormalities in membrane metabolism (Stanley et al 1994; Stanley et al 1995; Bertolino et al 1998; Auer et al 2001; O’Neill et al 2004; Tanaka et al 2006).

We report a case diagnosed as VEOS, with premorbid year-long psychopathological history, who showed atypical multivoxel proton magnetic resonance spectroscopy ( $\left.{ }^{1} \mathrm{H}-\mathrm{MRS}\right)$ findings, with normal brain and spine computer tomography (CT) and brain magnetic resonance (MR) images.

\section{Case report}

\section{The patient is a I5.3-year-old girl}

The psychodiagnostic assessment included semistructured interviews and rating scales administered to the patients and to their parents (Schedule for Affective Disorders and Schizophrenia for School-Age Children - Present and Lifetime Version, Child Behavior Checklist, Children Depression Rating Scale, Children Depression Inventory, Screen for Child Anxiety Related Disorders). The clinical and instrumental assessment comprised physical and neurological examinations, general laboratory investigation, and neuroimaging study.

\section{Family history}

The family belongs to the upper social class. The father shows obsessive personality traits, and was treated with paroxetine; the mother is affected by schizotypal personality disorder. The 10-year-old brother shows enuresis. The maternal grandfather is described as introvert and depressed; the maternal grandmother as intrusive and hypercritical. A paternal first cousin is affected by anorexia nervosa.

\section{Clinical history}

The patient was born by natural delivery at the eighth month of gestation, after a physiological pregnancy. At birth she evidenced cyanosis and mild respiratory distress. She showed mild motor developmental delay. From birth she showed poor eye contact, absence of smiling and weeping, severe difficulties in social skills, agitation bursts, and separation anxiety. She spent much of her time swinging herself. During primary school academic performance was inadequate, as was her relationship with peers. At age 9 she showed sleep disturbances, enuresis, and visual and auditory hallucinations. Her social withdrawal worsened over time, and was associated with bizarre and disorganized behavior. Academic performance also declined still further. She was started on psychotherapy. She came to our first observation at the age of 11 years. The patient appeared a chubby, clumsy, slow girl, dressed as an adult, with a hypomimic, and perplexed face. Eye contact was poor. Her speech was digressive, overlabored, and affected, with loosening of associations and altered prosody. She evidenced poor attention, while she showed good writing narrative abilities (she wrote long, fantastical histories). She reported visual and auditory hallucinations, and persecutory themes. She was diagnosed as suffering from paranoid schizophrenia (according to criteria from DSM-IV), and pharmacotherapy with olanzapine was started; psychotherapy continued. At age 13, she began to exhibit mood swings, aggression, and occasional explosive outbursts. Olanzapine was replaced with haloperidol, because of weight gain. She showed partial remission over the next 6 months: the visual hallucinations, and aggression disappeared; her behavior became more organized. At the age of 14.7 years she presented a psychiatry emergency: she felt herself to be constantly followed by someone; during the night she would often wake up hearing a man weeping, who asked her to help him; she heard an imperative, frightening male voice in her head, which told her to leave her room; during the day she saw scenes of blood. She reported a deep sense of confusion, anguish, thoughts of death and suicide, and a fear of hurting others. Lorazepam was added to haloperidol. She quickly showed a remission of the state of anguish, a significant reduction of the visual hallucinations, and a slight improvement of mood. At the last observation, at the age of 15.3 years, she showed affective flattening, sporadic auditory hallucinations, sexual disinhibition, bizarre behavior, and refusal to attend school.

\section{Neuroimaging study}

Brain and spine CT showed normal findings. Brain MR examination showed normal dimensions and morphology of cerebral ventricles and no altered signal of parenchyma. ${ }^{1} \mathrm{H}$-MRS was performed in the frontal regions and in the right semioval center, using the MR General Electric, Signa Lx and Mr/I Milwaukee 1.5 Tesla Unit. The ${ }^{1} \mathrm{H}-\mathrm{MRS}$ protocol consisted of multivoxel water suppressed spectra $(\mathrm{TR} / \mathrm{TE}=2100 / 96.4)$ with a voxel size of $20 \times 20 \times 15 \mathrm{~mm}$. ${ }^{1} \mathrm{H}-\mathrm{MRS}$ showed an elevated peak of lipids in both frontal regions (Figure 1a, b), with normal values for $\mathrm{N}$-acetylaspartate (NAA), creatine plus phosphocreatine ( $\mathrm{Cr}$ ), and choline (Cho), and $\mathrm{NAA} / \mathrm{Cr}$ and $\mathrm{Cho} / \mathrm{Cr}$ ratios. The findings in the right semioval center were all normal (Figure 2). The values were compared with the values obtained from a control group of 20 healthy patients aged 10 to 15 years who had undergone MRI for headache or epilepsy. In MRI of the control group 


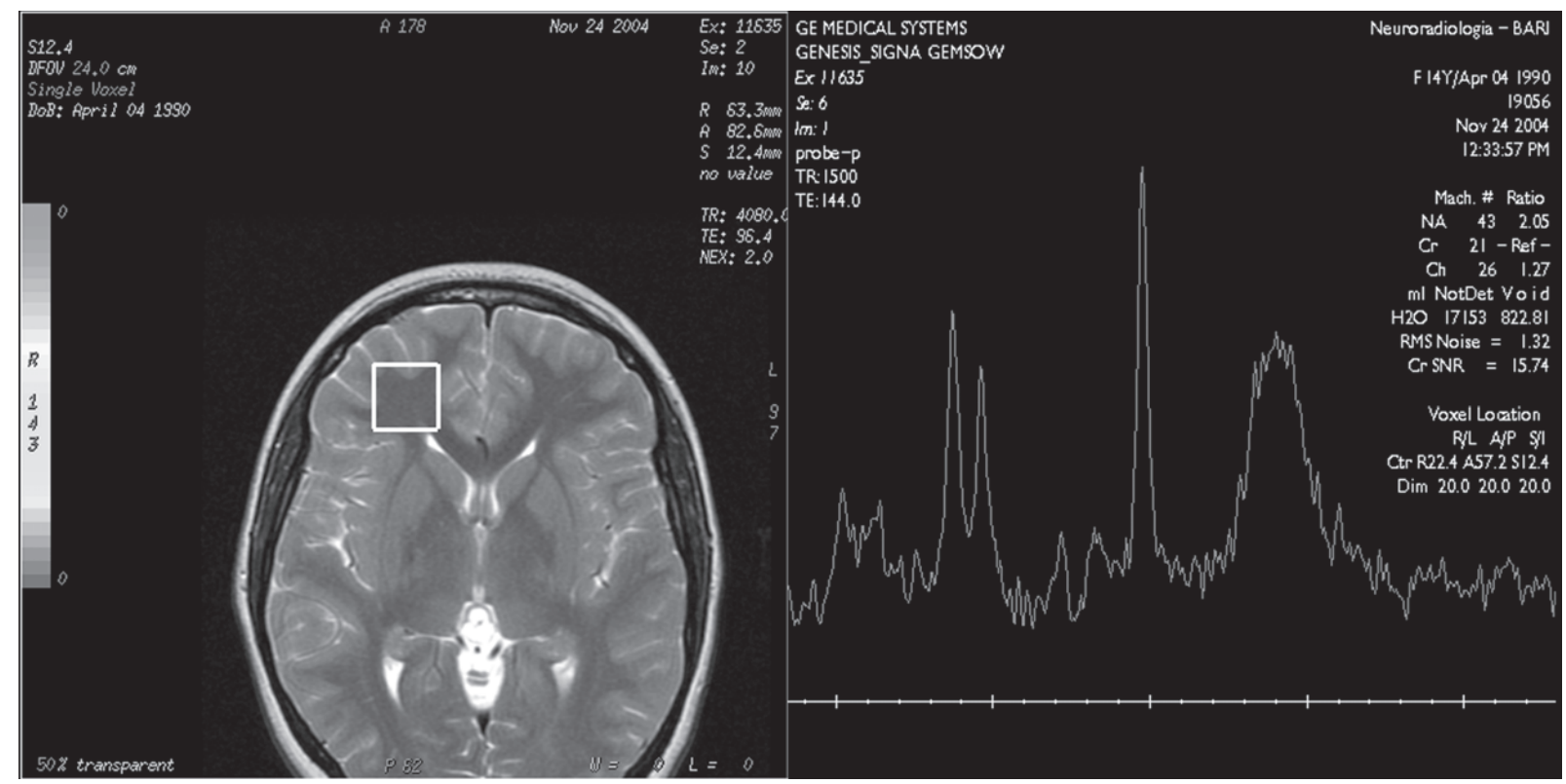

Figure Ia Multivoxel 'H-MRS (by GE Signa LX I,5 T):axial T2-weighted images (TE $96.4 \mathrm{~ms}$ ), multivoxel localization at the level of left frontal lobe. 'H-MRS spectra show an abnormal peak of lipids.

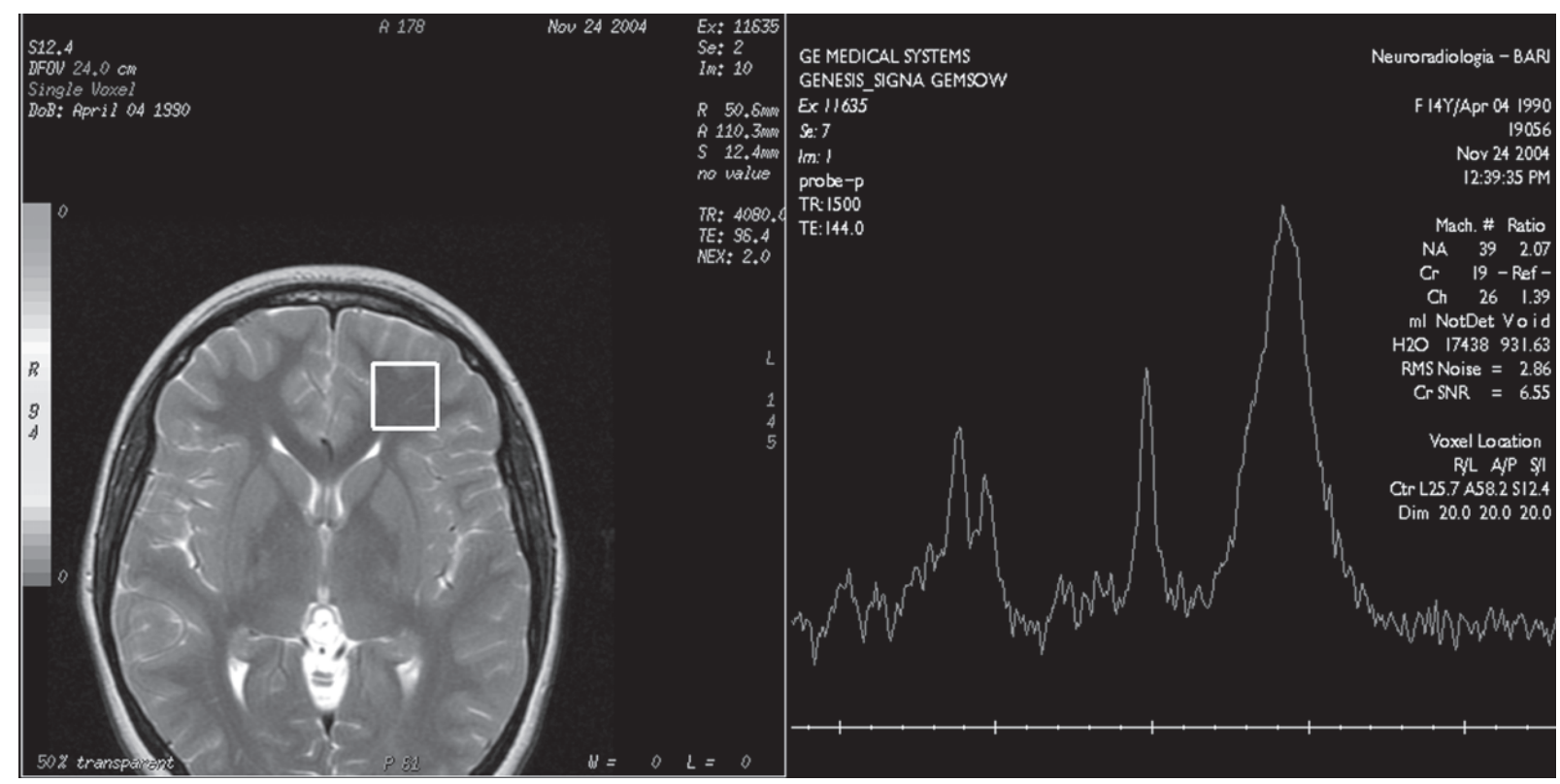

Figure Ib Multivoxel 'H-MRS (by GE Signa LX I,5 T): axial T2-weighted images (TE $96.4 \mathrm{~ms}$ ), multivoxel localization at the level of right frontal lobe. 'H-MRS spectra show a peak of lipids.

the conventional sequences did not show alterations of parenchyma and ventricles. The ${ }^{1} \mathrm{H}-\mathrm{MRS}$ (multivoxel water suppressed spectra TR/TE $=2100 / 26.4$ with voxel size of $20 \times 20 \times 15 \mathrm{~mm}$ ) showed only NAA, Cho, and Cr peaks at normal concentrations, and no peak of lipids.

\section{Discussion}

Although schizophrenia has been diagnosed in children, this disorder has received too little attention in the clinical and research literature. A greater frequency or severity of genetic and nongenetic risk factors could result in the early onset of schizophrenia; VEOS and EOS provide an opportunity to look for the relationships between genetic and environmental factors, neurodevelopmental abnormalities, and premorbid indicators of schizophrenia (Werry 1992; WHO 1992; Asarnow and Asarnow 1994; Russel 1994; Campbell et al 1997; Jacobsen and Rapoport 1998; Nicolson and Rapoport 1999; APA 2000; 


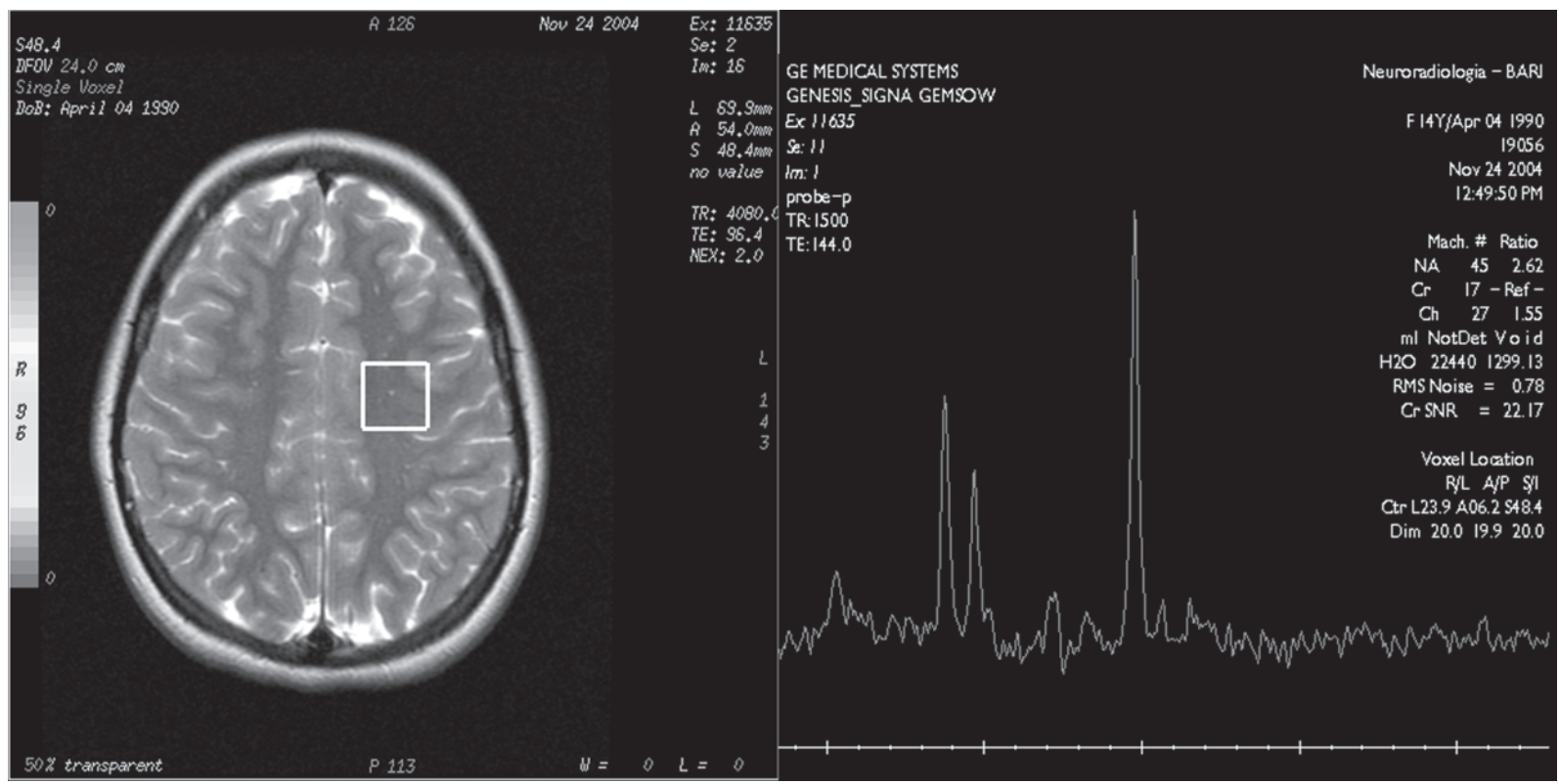

Figure 2 Multivoxel 'H-MRS (by GE Signa LX I,5 T): axial T2-weighted images (TE $96.4 \mathrm{~ms}$ ), multivoxel localization at the level of right semioval center. 'H-MRS spectra show normal values.

Nicolson et al 2000; Asarnow et al 2004; Bailly and de Chouly de Lenclave 2004).

Schizophrenia is actually considered to be a neurodevelopmental disorder: abnormalities in brain development and maturation seem to begin prenatally, but continue through childhood, and the changes observed over the time have consequences for neuronal circuitry and connectivity (Watkins et al 1988; Gourion et al 2004; Maki et al 2005). Recent research has focused on the premorbid indicators of schizophrenia, in order to improve the diagnostic and therapeutic interventions in the prodromal phases of the illness (Asarnow and Asarnow 1994; Russel 1994; Allen et al 2005; Maki et al 2005). In fact, early detection and precocious treatment of the first episode of schizophrenia could modify the outcome.

Premorbid abnormalities in academic, motor, language, and affective development are considered to be a neurodevelopmental marker unique to schizophrenia, social deterioration being a more general premorbid feature of severe affective and other psychotic illness (AlaghbandRad et al 1995; Allen et al 2005). In addition, several studies suggest that more pronounced early developmental premorbid abnormalities are usually associated with a poor outcome (Alaghband-Rad et al 1995). Although these early signs and prodromal symptoms can be defined retrospectively in patients who have developed schizophrenia, they still have to be confirmed as early predictors in prospective and longitudinal studies.
Recent studies underline the correlation of genetic, epigenetic, and environmental factors in the etiopathogenesis of schizophrenia, which might explain the heterogeneity of symptoms, course, and outcome (Petronis 2004). Molecular genetic studies have identified several chromosomal abnormalities, and evidence is accumulating in favor of several positional candidate genes (Petronis 2004). Familial studies have shown that parents of patients with VEOS/EOS have higher rates of schizophrenia and related spectrum disorders (schizoaffective disorder, schizotypal personality disorder, paranoid personality disorder) than parents of patients with adulthood-onset illness, and relatives of children and adolescents with attention deficit hyperactivity disorder (Asarnow et al 2001; Nicolson et al 2003). The parents of these patients also showed a higher rate of social isolation, introversion, suspiciousness, and hostility than parents of children with autism and attention deficit hyperactivity disorder (Asarnow et al 2001; Nicolson et al 2003).

In our patient, premorbid symptoms were present from birth, and signs of cognitive, emotional, and behavioral dysfunction were evident over the years. Despite these precocious signs, she was not observed medically until 9 years of age. Moreover, her parents showed several psychopathologies, also of the related spectrum disorders, suggesting a greater genetic vulnerability associated with acquired environmental factors. Our patient is particularly interesting because she provides a critical window into the inter-relationships between 
genetic predisposition, neurodevelopment, and premorbid indicators of schizophrenia.

The last two decades of neuroscience research have greatly expanded our understanding of the processes involved in the human brain's structural development and functional anatomy. There is evidence for structural and functional brain abnormalities in schizophrenia, suggesting abnormal local cell-energy metabolism and phospholipid membrane abnormalities (Stanley et al 1994, 1995; Bertolino et al 1998; Auer et al 2001; O’Neill et al 2004; Tanaka et al 2006). The principal findings of ${ }^{1} \mathrm{H}-\mathrm{MRS}$ in childhood-onset schizophrenia reveal abovenormal levels of $\mathrm{Cr}$ in the superior anterior cingulate; above-normal levels of Cho compounds in the superior anterior cingulate, frontal cortex, and caudate head; lower NAA in the thalamus; and decreased NAA/Cr ratio in the frontal cortex and hippocampus (Bertolino et al 1998; Auer et al 2001; O’Neill et al 2004; Tanaka et al 2006). The most consistent evidence for membrane phospholipid abnormalities in schizophrenia derives from phosphorus 31 magnetic resonance spectroscopy studies, indicating reduced membrane phospholipid precursors in early phases of schizophrenia (Stanley et al 1995; Auer et al 2001). The decreased synthesis and increased breakdown of membrane phospholipids might modify the structure and function of the neuronal membrane, microenvironment, receptors, ion channels, and enzymes, resulting in an altered neurotransmission (Fenton et al 2000). Peaks of lipids are reported in adolescents with a bipolar disorder (Castillo et al 2000), and have never been reported in ${ }^{1} \mathrm{H}-\mathrm{MRS}$ study in cases with VEOS/EOS. Follow-up studies are needed to examine the predictive value of these measures.

Our patient showed unique ${ }^{1} \mathrm{H}-\mathrm{MRS}$ findings. We could hypothesize that the ${ }^{1} \mathrm{H}-\mathrm{MRS}$ findings might be a precocious alteration leading to other abnormalities over time, consistent with a neurodevelopmental model of this illness, but we cannot exclude the possibility that these findings are the result of a progression of the illness, consistent with a neurodegenerative model.

Additional clinical studies on childhood schizophrenia are required to determine the very early signs and symptoms that indicate a VEOS/EOS, and whether the premorbid period should not already be regarded as illness, and treated accordingly. Further delineation of biological markers is necessary to identity the etiopathogenesis of EOS/VEOS. In particular, ${ }^{1} \mathrm{H}-\mathrm{MRS}$ is a noninvasive investigative technique that could provide useful insights into the biochemical basis of schizophrenia.

\section{Disclosure}

The authors report no conflicts of interest in this work.

\section{References}

Alaghband-Rad J, McKenna K, Gordon CT, et al. 1995. Childhood-onset schizophrenia: the severity of premorbid course. J Am Acad Child Adolesc Psychiatry, 34:1273-83.

Allen DN, Frantom LV, Strauss GP, et al. 2005. Differential patterns of premorbid academic and social deterioration in patients with schizophrenia. Schizophr Res, 75:389-97.

[APA] American Psychiatric Association. 2000. DSM IV-TR Diagnostic and Statistical Manual of Mental Disorders, Fourth Edition, Text Revision. Masson.

Asarnow RF, Asarnow JR. 1994. Childhood-onset schizophrenia: Editors' introduction. Schizophr Bull, 20:591-7.

Asarnow RF, Nuechterlein KH, Fogelson DL, et al. 2001. Schizophrenia and schizophrenia-spectrum personality disorders in the first-degree relatives of children with schizophrenia: the UCLA family study. Arch Gen Psychiatry, 58:581-8.

Asarnow JR, Tompson MC, McGrath EP. 2004. Annotation: childhoodonset schizophrenia: clinical and treatment issues. $J$ Child Psychol Psychiatry, 45(2):180-94.

Auer DP, Wilke M, Grabner A, et al. 2001. Reduced NAA in the thalamus and altered membrane and glial metabolism in schizophrenic patients detected by $1 \mathrm{H}$ MRS and tissue segmentation. Schizophr Res, 52:87-99.

Bailly D, de Chouly de Lenclave MB. 2004. A rare and not very studied disorder: childood-onset schizophrenia. A case report. Encephale, 30:540-7.

Bertolino A, Kumra S, Callicott JH, et al. 1998. Common pattern of cortical pathology in childhood-onset and adult-onset schizophrenia as identified by proton magnetic resonance spectroscopic imaging. Am J Psychiatry, 155:1376-83.

Campbell M, Armenteros JL, Spencer EK. 1997. Schizophrenia and psychotic disorders. In: Wiener JM (ed). Textbook of Child and Adolescent Psychiatry. Washington, DC: American Academy of Child and Adolescent Psychiatry.

Castillo M, Kwock L, Courvoisie H, et al. 2000. Proton MR spectroscopy in children with bipolar affective disorder: preliminary observations. Am J Neuroradiol, 21:832-8.

Fenton WS, Hibbeln J, Knable M. 2000. Essential fatty acids, lipid membrane abnormlities and the diagnosis and treatment of schizophrenia. Biol Psychiatry, 47:8-21.

Gourion D, Gourevitch R, Leprovost JB, et al. 2004. Neurodevelopmental hypothesis in schizophrenia. Encephale, 30:109-18.

Jacobsen LK, Rapoport JL. 1998. Childhood-onset schizophrenia: implications of clinical and neurobiological research. J Child Psychol Psychiatry, 38:607-712.

Maki P, Veijola J, Jones PB, et al. 2005. Predictors of schizophrenia: a review. Br Med Bull, 9(73):1-15.

Nicolson R, Rapoport JL. 1999. Childhood-onset schizophrenia: rare but worth studying. Biol Psychiatry, 46:1418-28.

Nicolson R, Lenane M, Hamburger SD, et al. 2000. Lessons from childhood-onset schizophrenia. Brain Res Rev, 31:147-56.

Nicolson R, Brookner FB, Lenane, et al. 2003. Parental schizophrenia spectrum disorders in childhood-onset and adult-onset schizophrenia. Am J Psychiatry, 160:490-5.

O’Neill J, Levitt J, Caplan R, et al. 2004. 1H-MRSI evidence of metabolic abnormalities in childhood-onset schizophrenia. Neuroimage, 1:1781-9.

Petronis A. 2004. The origin of schizophrenia: genetic thesis, epigenetic antithesis, and resolving synthesis. Biol Psychiatry, 55:965-70.

Russell AT. 1994. The clinical presentation of childhood-onset schizophrenia. Schizophr Bull, 20:631-46.

Stanley JA, Williamson PC, Drost DJ, et al. 1994. Membrane phospholipids metabolism and schizophrenia: an in vivo 31P-MR spectroscopy study. Schizophr Res, 13:209-15. 
Stanley JA, Williamson PC, Drost DJ, et al. 1995. An in vivo study of the prefrontal cortex of schizophrenic patients at different stages of illness via phosphorus magnetic resonance spectroscopy. Arch Gen Psychiatry, 52:399-406.

Tanaka Y, Obata T, Sassa T, et al. 2006. Quantitative magnetic resonance spectrosopy of schizophrenia: relationship between decreased $\mathrm{N}$-acetylaspartate and frontal lobe dysfunction. Psychiatry Clin Neurosci, 60:365-72.
Watkins JM, Asarnow RF, Tanguay PE. 1988. Symptom development in childhood onset schizophrenia. J Child Psychol Psychiatry, 29:865-78.

Werry JS. 1992. Child and adolescent (early onset) schizophrenia: a review in light of DSM-III-R. Autism Dev Disord, 22:601-24.

[WHO] World Health Organization. 1992. The ICD-10 Classification of Mental and Behavioral Disorder: Clinical descriptions and Diagnostic Guidelines. 10th revision. Geneva (WHO). 
